This PDF is a selection from an out-of-print volume from the National Bureau of Economic Research

Volume Title: Evaluation of Econometric Models

Volume Author/Editor: Jan Kmenta and James B. Ramsey, eds.

Volume Publisher: Academic Press

Volume ISBN: 978-0-12-416550-2

Volume URL: http://www.nber.org/books/kmen80-1

Publication Date: 1980

Chapter Title: Model Construction and Evaluation When Theoretical Knowledge Is Scarce

Chapter Author: Herman Wold

Chapter URL: http://www.nber.org/chapters/c11693

Chapter pages in book: (p. 47 - 74) 


\title{
Model Construction and Evaluation When Theoretical Knowledge Is Scarce
}

\author{
Theory and Application of \\ Partial Least Squares
}

HERMAN WOLD

DEPARTMENT OF STATISTICS

UNIVERSITY OF UPPSALA

UPPSALA, SWEDEN

\section{INTRODUCTION}

During the past decade many econometricians have come to realize that the use of much of the contemporary set of rigorous tools of statistical analysis is not applicable in all circumstances, mainly because the knowledge requirements of the investigator are far greater than are available. For example, a typical regression problem will require the researcher to know the relevant variables in the regression, its functional form, whether or not the observed relationship can be identified with the theoretical concept, and knowledge of the relevant distributions up to estimable parameter values.

Unfortunately, there are few topics in economics where these assumptions are tenable, at least in full. There are two areas of knowledge deficiency. First, in many topics in economics our theories are merely prescriptions of a likely list of causal variables for some specified set of effect variables. Second, our knowledge of the statistical distributional properties of the relevant variables is even less complete. A related empirical difficulty is that usually the variables of direct interest cannot be observed and one must rely on 
indicator (marker) variables which are assumed to have some degree of association with the variables of theoretical interest. Under such circumstances to proceed along conventional lines with numerous heroic assumptions is inferentially hazardous. Another less ambitious but less knowledge sensitive approach is needed. Two items are required: a less knowledge intensive formulation of theory and a robust statistical procedure for drawing inferences when one is ignorant about the relevant statistical distributions. The former requirement can be met by the development of what has come to be known in the sociological literature as "path models." The latter requirement is met by the use of partial least squares.

\section{Path Models: Their Use in the Modeling OF RUDIMENTARY THEORIES}

Path models and their use are best explained in terms of an example. Consider the inferential problem faced by Adelman \& Morriss (1967, 1973), who wished to learn something about the "causes of economic growth" in noncommunist developing countries. Seventy-four countries provided observations on aspects of economic growth together with data on 41 indicators of economic, social, and political factors such as rate of growth of real per capita income, abundance of natural resources, extent of social mobility, and degree of competitiveness of political parties; see Table 1.

The initial analysis of these data used principal components to classify all 74 countries into three phases of economic growth: low, sporadic or abortive, and high; see Fig. 1a. Adelman and Morriss felt that their investigation of the causes of differential growth rates would be supported by little theoretical insight beyond a list of potentially useful indicators of growth and of the causes of growth differentials. Further, no detailed assumptions about statistical distributions could be made.

In a later investigation based on the same data, Adelman et al. (1975a) designed a path model to explain economic growth in terms of economic, social, and political conditions. Whatever conceptual-theoretical insight there is in the model is illustrated diagrammatically by its arrow scheme; see Fig. 1b. The elementary notions are these: Social conditions affect both political conditions and economic growth rates. Economic levels also affect both political conditions and economic growth rates. Finally, the political conditions themselves clearly affect growth rates. Apart from these general statements about the four categories of the model-economic levels, social

${ }^{1}$ Blalock $(1964,1971)$. 
and political conditions, economic growth rates - there is the pivotal question of what observable variables can be used as suitable markers or indicators for these categories. The 39 observables that are selected as indicators for the four blocks of the model are listed in Table 1 . With respect to the causal-predictive purposes of the model, one would like to obtain some measure of the relative effect of, say, economic levels and political conditions, on growth rates. The development of path models enables one to provide initial, albeit tentative, answers to these questions.

\section{TABLE 1}

The Four Blocks of Indicator Variables in the Adelman Model ${ }^{a}$

\begin{tabular}{|c|c|}
\hline Block No. 1 & Block No. 3 \\
\hline (E) Indicators of economic Levels & (P) Indicators of political conditions \\
\hline 1. Level of infrastructure & 1 Degree of administrative efficiency \\
\hline 2 Level of industry & 2 Degree of centralization of political \\
\hline Level of agriculture & power \\
\hline Dualism & 3 Democratic tradition \\
\hline Per capita GNP & 4 Degree of freedom of the press \\
\hline Structure of trade & 5 Predominant basis of the political \\
\hline Abundance of natural resources & party system \\
\hline & 6 Factionalization of parties \\
\hline & 7 Extent of political stability \\
\hline & 8 Extent of leadership commitment \\
\hline & 9 Strength of labor movement \\
\hline & 10 Political strength of the traditional elite \\
\hline & 11 Political strength of the military \\
\hline Block No. 2 & Block No. 4 \\
\hline (S) Indicators of social conditions & (G) Indicators of economic growth \\
\hline 1 Size of traditional agricul tural sector & 1 Rate of change in per capita GNP \\
\hline 2 Modernization of outlook & 2 Investment rate \\
\hline 3 Size of middle class & 3 Improvement in taxation system \\
\hline 4 Extent of social mobility & 4 Improvement in degree of \\
\hline 5 Extent of literacy & industrialization \\
\hline 6 Extent of mass communication & 5 Improvement in financial institutions \\
\hline Degree of social tension & 6 Improvement in agriculture \\
\hline National integration & 7 Improvement in infrastructure \\
\hline Ethnic homogeneity & 8 Rate of improvement of human \\
\hline Crude fertility rate & resources \\
\hline Urbanization & \\
\hline Agricultural organization & \\
\hline 13 Social organization & \\
\hline
\end{tabular}

a Adelman et al. (1975a). Two of the 41 indicators in the data bank were dropped in the soft model reported here. 


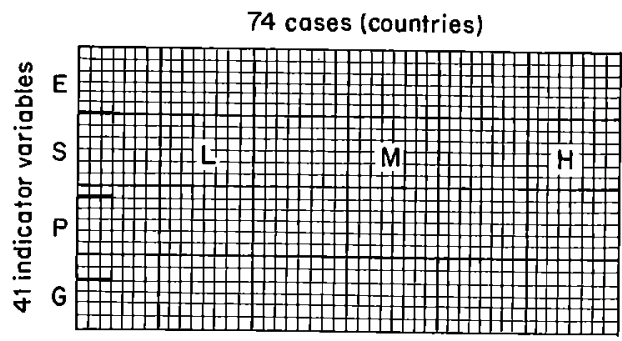

(a)

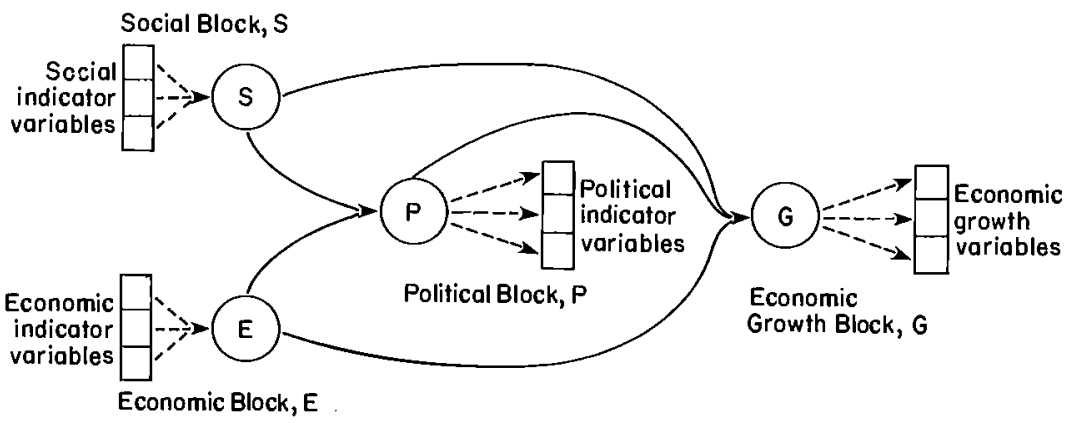

(b)

Fig. 1. Data matrix and arrow scheme for Adelman's model. (a) Classification of 74 countries : Low (L), Sporadic $(\mathrm{M})$ and High $(\mathrm{H})$ degrees of economic growth, using 41 indicators : Economic levels (E), social indicators (S), political indicators $(P)$, economic growth rates $(G)$. Ref. Adelman \& Morriss $(1967$; 1973) (b) Four-block model designed by Adelman et al. (1975a) : Economic levels and Social conditions influence the Political conditions; these three blocks influence the Economic growth rates.

Path models with manifest (directly observed) variables have been a principal tool in econometrics since the 1930s. Path models with latent (indirectly observed) variables were initiated in sociology about $1960 .^{2}$ Conceptually, the transition from manifest to latent variables in path modeling borrowed its rationale from the classical models of factor analysis in psychology. The transition to latent variables opened a new field of interest in statistical inference. Statistical methods for estimating the parameters of path models with latent variables have been developed by K. G. Jöreskog (1970, 1973, 1978, 1980), using his LISREL versions of the ML (Maximum Likelihood) approach, and by H. Wold (1974, 1975a,b,c,d, 1977a,b, 1978a,b, 1980b) using PLS (Partial Least Squares) estimation.

\footnotetext{
${ }^{2}$ Cf. Duncan (1966).
} 
The present paper gives a brief exposition of the PLS approach to path models with latent variables. The next section begins with a broad overall view of the PLS procedure.

\section{The PLS Approach to Path Models with Latent VARIABles: AN OVERVIEW OF ENDs and MEANS}

The PLS approach to path models with latent variables is primarily intended for multidisciplinary and other applications where the problems explored are complex and theoretical knowledge is scarce. Three characteristics are involved: (a) causal-predictive analysis, (b) complexity of the problems explored, and (c) scarcity of prior theoretical knowledge.

In this section the emphasis is on how these characteristics are reflected in each of the four phases of the construction of a PLS path model with latent variables: theoretical specification, estimation, testing, and evaluation.

\section{Theoretical Definition of the Model}

The design of a PLS path model with latent variables is indicated by its arrow scheme and its block structure. The arrow scheme illustrates basic features of the model. The manifest variables are grouped into blocks. In each block the manifest variables are assumed to be indicators of a latent variable (LV). The LVs, which the structural units of the model, are interrelated by inner relations. The formal relationships between the LVs and their indicators constitute the block structure. The arrows indicate channels of information in the model.

The specification of the block structure and the inner relations constitutes the theoretical definition of the model. The residuals for the estimated inner relations and relationships between LVs and indicators are marked by arrow heads in Fig. 1b. A feature of the model, which is not explicitly illustrated by the arrow scheme, is the specification of the causal-predictive relations. As corollaries of the specification of the block structure and the inner relations, they provide causal-predictive inferences on the indicators of those LVs that are effect variables in the inner relations, namely, inferences in terms of indicators and/or LVs of the corresponding explanatory variables. The block structure, the inner relations, and the causal-predictive relations are called the structural relations of the model.

\section{Estimation of the Model}

The estimation procedure for a PLS model with LVs has four characteristic features. Each LV is estimated as a weighted aggregate of its indicators. The weights of the indicators in each aggregate are determined by the 
weight relations of the various blocks. The model builder has the option to choose among different modes-A, B, or C-for the design of the weight relations. The selection of estimation mode is guided by the subject matter of the model. The estimation proceeds in three stages. First, an iterative procedure estimates the weights and the LVs. Second, the LVs estimated in the first stage provide regressors for estimating the other unknown coefficients of the model by OLS (Ordinary Least Squares) regressions. It is important to note that in the third stage the location parameters are estimated.

\section{Testing the Model}

With respect to the testing and evaluation of the model, classical methods such as confidence intervals and goodness of fit, which are based on distributional properties of the observables, are not available because of the scarcity of theoretical knowledge. PLS modeling instead uses LS (Least Squares)-oriented but distribution-free methods. This parting of the ways is technical rather than real, for ML aims for optimal accuracy but PLS for consistency. Under regular conditions ML and PLS estimates are co-consistent, so that there is no substantial difference between the two set of estimates.

Two types of statistical tests have been developed for PLS path models with LVs. An overall test of the model is provided by the predictive significance of the model. The procedure uses an adaptation of the Stone-Geisser (1974) cross-validation method. The robustness of the various parameter estimates is tested by random perturbation of the data (see Adelman et al. $(1975 b))$.

\section{Model Formulation When Theoretical Knowledge Is Scarce}

We begin our discussion of the formulation of soft models with some notational details. In our general formulas for the basic design of PLS path models with LVs we shall use the following notation. $J$ denotes the number of blocks and $K_{j}$ the number of indicators in the $j$ th block. The manifest variables are denoted by

$$
x_{j k}: \quad j=1, \ldots, J ; k=1, \ldots, K_{j}
$$

and the latent variables by

$$
\xi_{j}: \quad j=1, \ldots, J
$$


The values taken by the manifest and latent variables, which are also known as "case values," are denoted by

$$
x_{j k n}, \xi_{j n}: \quad j=1, \ldots, J, \quad k=1, \ldots, K_{j}, \quad n=1, \ldots, N .
$$

As is customary, we denote theoretical entities by Greek letters and the corresponding estimates by the appropriate Roman letters. The data bank of Adelman and Morriss $(1967,1973)$ has $N=74$ observations on 41 indicators of economic, social, and political conditions. Adelman's model, given by the arrow scheme shown in Fig. $1 \mathrm{~b}$, groups the observables into $J=4$ blocks, with $K_{1}=7$ indicators of economic levels, $\mathrm{E} ; K_{2}=13$ indicators of social conditions, $\mathbf{S} ; K_{3}=11$ indicators of political conditions, $\mathbf{P}$; and $K_{4}=8$ indicators of economic growth, G; see Table 1 .

\subsection{The Arrow Scheme}

The arrow scheme groups the observables-that is, the manifest variables-into blocks of indicators, designates the hypothetical latent variables, and shows the relationships between the LVs; these are assumed to form a causal chain system. ${ }^{3}$ The "outer" arrows between each LV and its indicators serve to illustrate the optional choice of estimation modes A, B, or $\mathrm{C}$, which will be discussed in Section 2. Depending upon their position in the causal chain system, the blocks and their corresponding latent variables are either exogenous or endogenous. In Adelman's model the two blocks and LVs of economic levels and social conditions are exogenous, the two blocks and LVs of political conditions and economic growth rates are endogenous.

\subsection{The Block Structure}

In the basic design of PLS path models with LVs, the block structure is assumed to be linear:

$$
x_{j k}=\mu_{j k}+\pi_{j k} \xi_{j}+v_{j k}, \quad j=1, \ldots, K, \quad k=1, \ldots, K_{j},
$$

where $\xi_{j}$ is the LV of the $j$ th block, $\pi_{j k}$ is the loading of the $k$ th indicator $x_{j k}$ in the $j$ th block, the $\mu$ s are location parameters and the $v$ s are residuals in the block structure.

${ }^{3}$ As to the general rationale of causal chain systems and interdependent systems, PLS modeling adheres to the exposition in Mosbaek \& $\mathrm{H}$. Wold (1970). 
In each relation in (4), the systematic part is assumed to be the conditional expectation of the indicator when the latent variable is given-that is,

$$
E\left(x_{j k} \mid \xi_{j}\right)=\mu_{j k}+\pi_{j k} \xi_{j}, \quad j=1, \ldots, J, \quad k=1, \ldots, K_{j}{ }^{4}
$$

The "predictor" assumption in (5) implies that each residual in each equation has a conditional expectation of zero and is uncorrelated with the latent variable occurring in that equation. Since both $\pi_{j k}$ and $\xi_{j}$ are unknown in the block structure in (4), some standardization of scale is necessary in order to avoid ambiguity. The choice of standardization does not affect the substantive results of the statistical analysis. In PLS path models all LVs are standardized so as to have unit variance:

$$
\operatorname{var}\left(\xi_{j}\right)=1, \quad j=1, \ldots, J
$$

It is a fundamental principle in PLS path modeling that the information between the blocks and the ensuing causal-predictive inference is conveyed through the latent variables. Accordingly, it is assumed that the latent variables are, in general, intercorrelated, say,

$$
r\left(\xi_{i}, \xi_{j}\right)=\rho_{i j}, \quad i, j=1, \ldots, J,
$$

whereas the residuals of any block are assumed to be uncorrelated with the residuals of other blocks and with all latent variables:

$$
\begin{gathered}
r\left(v_{i h}, v_{j k}\right)=r\left(v_{i h}, \xi_{i}\right)=r\left(v_{i h}, \xi_{j}\right)=0, \\
i \neq j, \quad i, j=1, \ldots, J, \quad h=1, \ldots, K_{i}, \quad k=1, \ldots, K_{j} .
\end{gathered}
$$

In the basic model design it is assumed, furthermore, that the residuals are mutually uncorrelated within blocks:

$$
r\left(v_{j h}, v_{j k}\right)=0, \quad j=1, \ldots, J, \quad h \neq k=1, \ldots, K_{j} .
$$

Formally, the block structure as specified by Eqs. (4)-(9) is the same for all PLS models. What differs between models is the specified number of blocks and the number of indicators in each block.

Just as the scales of the latent variables $\xi_{j}$ are ambiguous in the block structure, so also are the signs of $\xi_{j}$ and $\pi_{j k}$. Clearly, a change in the sign in the $j$ th latent variable will change correspondingly the signs of all its loadings $\pi_{j k}, k=1,2, \ldots, K_{j}$. The choice of sign is inherent in the subject matter of the model. The model builder defines the manifest variables $x_{j k}$ so that their signs are in accordance with the nature of the postulated latent variable $\xi_{j}$. The sign of $\xi_{j}$ then determines the signs of the loading $\pi_{j k}$ in the block

4 The "predictor" specification (5) provides the basis for causal-predictive inferences from (4) and at the same time for consistent estimation of the parameters by OLS regression, cf. $\mathrm{H}$. Wold (1963). 
structure in (4). Hence, when the loadings are estimated, their more or less complete agreement with the postulated signs provides a partial test of hypotheses.

For example, in the first block of Adelman's model all indicators are assumed to be positively related to the LV for level of economic development, $\pi_{1, k}>0, j=1, k=1,2, \ldots, 7$. In the second block, the size of the traditional agricultural sector is negatively related to the LV for social conditions, $\pi_{2,1}<0, j=2, k=1$.

\subsection{The InNer Relations}

The LVs of a PLS path model are related by a path of "inner" relations, which are linear and form a causal chain system. Using subscripts $j_{*}$ for the endogenous variables - say, $H$ in number - the inner relations are denoted by

$$
\xi_{j_{*}}=\alpha_{j_{*}}+\beta_{\left(j_{*} \xi_{\left(j_{*}\right)}\right.}+\varepsilon_{j_{*}}, \quad j_{*}=j_{1}, \ldots, j_{H},
$$

and are specified by the conditional expectations

$$
E\left(\xi_{j_{*}} \mid \xi_{\left(j_{*}\right)}\right)=\alpha_{j_{*}}+\beta_{\left(j_{\star}\right)} \xi_{\left(j_{*}\right)}, \quad j_{*}=j_{1}, \ldots, j_{H},
$$

where $\xi_{\left(j_{x}\right)}$ denotes the column vector of the latent variables that appear as regressors with nonzero coefficients in the $j_{*}$ th equation as shown in (10) and $\beta_{\left(j_{*}\right)}$ is the row vector of the corresponding coefficients. Also, let $\xi_{j_{* *}}$ denote those latent variables with which $\xi_{j}$ is directly connected, say,

$$
j_{* *}=i_{j 1}, \ldots, i_{j q},
$$

with $i_{j a} \neq j$ and $a=1, \ldots, q$, where $q=q(i)$ and varies with $i$. For example, Adelman's model has two inner relations:

$$
\begin{aligned}
& \xi_{3}=\alpha_{3}+\beta_{31} \xi_{1}+\beta_{32} \xi_{2}+\varepsilon_{3}, \\
& \xi_{4}=\alpha_{4}+\beta_{41} \xi_{1}+\beta_{42} \xi_{2}+\beta_{43} \xi_{3}+\varepsilon_{4} .
\end{aligned}
$$

The conditional mean of Eq. (13) is

$$
E\left(\xi_{3} \mid \xi_{1}, \xi_{2}\right)=\alpha_{3}+\beta_{31} \xi_{1}+\beta_{32} \xi_{2}
$$

and similarly for Eq. (14). In terms of the subscript notation in Eqs. (10) and (12), we have

$$
\begin{aligned}
& 3_{*}=1,2, \quad 4_{*}=1,2,3, \\
& 1_{* *}=3,4, \quad 2_{* *}=3,4 \quad 3_{* *}=1,2,4, \quad 4_{* *}=1,2,3,
\end{aligned}
$$

where the formula for $1_{* *}$ indicates that LV 1 (economic block) is directly related to LVs 3 and 4 , the political block and the economic growth block, respectively. See Fig. 1b. 


\subsection{The Causal-Predictive Relations}

For the indicators of any endogenous LV the PLS approach to path models with LVs provides causal-predictive relations by Substitutive Elimination of the Latent Variable (SELV) from the corresponding block structure (4) and inner relation (10). Hence in the basic design, the causalpredictive relations read

$$
x_{j_{x^{k}}}=\mu_{j_{j_{k} k}}+\pi_{j_{k^{k}} \alpha_{j_{*}}}+\pi_{j_{*} k} \beta_{\left(j_{*}\right)} \xi_{\left(j_{*}\right)}+v_{j_{*} k}
$$

and the prediction errors are given by

$$
v_{j_{*} k}=v_{j_{*} k}+\pi_{j_{k} k} \varepsilon_{j_{*}}, \quad j_{*}=j_{1}, \ldots, j_{H}, \quad k=1, \ldots, K_{j_{*}} .
$$

In Adelman's model, the third and fourth blocks are endogenous, and so the PLS approach gives causal-predictive relations for the indicators of these blocks, namely,

$$
\begin{aligned}
x_{3 k}= & \mu_{3 k}+\pi_{3 k} \alpha_{3}+\pi_{3 k}\left(\beta_{31} \xi_{1}+\beta_{32} \xi_{2}\right)+v_{3 k}, \\
& k=1, \ldots, 11, \\
x_{4 k}= & \mu_{4 k}+\pi_{4 k} \alpha_{4}+\pi_{4 k}\left(\beta_{41} \xi_{1}+\beta_{42} \xi_{2}+\beta_{43} \xi_{3}\right)+v_{4 k}, \\
& k=1, \ldots, 8,
\end{aligned}
$$

with prediction errors given by

$$
v_{3 k}=v_{3 k}+\pi_{3 k} \varepsilon_{3}, \quad v_{4 k}=v_{4 k}+\pi_{4 k} \varepsilon_{4} .
$$

\section{Model Fitting and Parameter Estimation with PLS}

Proceeding to the PLS estimation of path models with LVs, we shall first give a formal exposition of the procedure and then discuss its rationale. PLS estimation can use the observed data defined in (1.3) either in terms of raw data or cross-product data. The estimates are numerically the same except for rounding-off errors. We shall set forth the case of raw data input. Postponing the estimation of the location parameters, we assume that all observables are standardized to zero mean, say,

$$
x_{j k n}^{\prime}=x_{j k n}-\bar{x}_{j k}, \quad j=1, \ldots, J, \quad k=1, \ldots, K_{j}, \quad n=1, \ldots, N .
$$

Until further notice, furthermore, we shall drop the prime superscripts, denoting the standardized observables as before by

$x_{j k n} \quad$ now with $\bar{x}_{j k}=0, \quad j=1, \ldots, J, \quad k=1, \ldots, K_{j}, \quad n=1, \ldots, N$. 
Each LV is to be estimated as a weighted aggregate of its indicators; the first stage of the estimation process estimates the LVs and the requisite sets of weights for the indicators.

If we denote the estimate of $\xi_{j}$ by $X_{j}$, with case values $X_{j n}$, the aggregate formula, which is the same for all LVs in a PLS path model, is

$$
X_{j n}=f_{J} \sum_{k}\left(w_{j k} x_{j k n}\right), \quad j=1, \ldots, J, \quad k=1, \ldots, K_{j}, \quad n=1, \ldots, N,
$$

where $f_{j}$ is a scalar, $w_{j k}$ are the weights, and $x_{j k n}$ are the case values for the manifest variables. Since $\xi_{j}$ is standardized to unit variance, $f_{j}$ is determined by the requirement that $X_{j n}$ have unit variance over the $N$ cases, i.e.,

$$
f_{j}= \pm\left\{\frac{1}{N} \sum_{n}\left(\sum_{k}\left(w_{j k} x_{j k n}\right)^{2}\right)\right\}^{-1 / 2}, \quad j=1, \ldots, J .
$$

The weight relations serve to determine the weights $w_{j k}$ in the PLS estimation of Eq. (3) for each $\mathrm{LV} \xi_{j}$ as a weighted aggregate of its indicators $x_{j k}$.

By the inner relations of the model, there is an exchange of information between LV $\xi_{j}$ of the $j$ th block and those LVs $\xi_{j_{k *}}$ with which $\xi_{j}$ is directly connected in the arrow scheme. Hence the weight relations are designed to provide ancillary information on the weights $w_{j k}$ from these same $\xi_{j_{k s}} \mathrm{~s}$, using OLS regressions for the purpose.

For each block, the model builder has the option to choose between two modes of weight relations, say A or B. We note in advance that both modes involve a sum of those LVs with which the LV of the block is directly connected in the arrow scheme, a sum with sign factors $(+1$ or -1$)$ denoted by $s_{j, j_{* \star}}$ and defined by

$$
s_{j, j_{* *}}=\operatorname{sgn} r\left(X_{j}, X_{j_{*}}\right), \quad j=1, \ldots, J, j_{* *}=i_{j 1}, \ldots, i_{j q},
$$

where the subscripts $j_{* *}$ were defined in Eq. (1.12). Modes A and B for the weight relations are defined as follows.

\subsection{Mode A. Simple OLS Regressions With}

THE INDICATORS AS REGRESSANDS

$$
\begin{aligned}
x_{j k}=w_{j k}\left(s_{j, i_{j 1}} X_{i_{j 1}}+s_{j, i_{j 2}} X_{i_{j 2}}+\cdots+s_{j, i_{j q}} X_{i_{j q}}\right) & +d_{j k} \\
k & =1, \ldots, K_{j},
\end{aligned}
$$

where $x_{j k}$ are the manifest variables, $X_{i_{j a}}$ are the predicted values for the LVs, $w_{j k}$ are the weights to be estimated, and $d_{j k}$ are the residuals. Here and in Eq. (7) the regressions are over $n=1, \ldots, N$; for simplicity, we have dropped the subscript $n$ in the manifest variables, the LVs, and the residuals. 


\subsection{Mode B. Multiple OLS Regression with} THE INDICATORS AS REGRESSORS

$$
s_{j, i_{j 1}} X_{i_{j 1}}+s_{j, i_{j 2}} X_{i_{j 2}}+\cdots+s_{j, i_{j q}} X_{i_{j q}}=\sum_{k}\left(w_{j k} x_{j k}\right)+d_{j} .
$$

The arrow scheme illustrates the weight relations mode A or B by "outer" arrows between the LVs and their indicators in the various blocks; see Fig. 1b. The simple OLS regressions of mode A are illustrated by as many arrows directed outwards from the LV to its indicators. The multiple OLS regression of mode $\mathrm{B}$ is illustrated by a bundle of arrows directed inwards from the indicators to the LV. In the arrow scheme of a PLS path model, the "outer" arrow heads illustrate the residuals of the corresponding weight relations in (6) or (7).

We shall say that the estimation is mode $C$ if the model builder makes combined use of modes A and B, each of the two modes being used for at least one block. The following rule of thumb is offered for the choice between $A$ and $B$ : If a block represents an exogenous $L V$, use B, otherwise, use A.

When the model builder has settled the choice of mode A or B for determining the weight relations in the various blocks of the model, the iterative procedure of estimation of LVs and indicator weights is performed by alternating between the weighted aggregates shown in Eqs. (3) and (4) and the weight relations shown in Eqs. (5)-(7).

If we write $r=1,2, \ldots$ for the consecutive steps in the iterative procedure and mark the proxy estimates obtained in steps $r$ and $(r+1)$ by the superscripts (') and ("), Eqs. (3)-(7) give the values of the LVs and of the weights in step $(r+1)$ in terms of the values obtained in step $r$ as follows:

$$
\begin{gathered}
X_{j n}^{\prime \prime}=f_{j}^{\prime \prime} \sum_{k}\left(w_{j k}^{\prime} x_{j k n}\right), \quad j=1, \ldots, J, \quad n=1, \ldots, N \\
f_{j}^{\prime \prime}= \pm\left\{\frac{1}{N} \sum_{n}\left[\sum_{k}\left(w_{j k}^{\prime} x_{j k n}\right)^{2}\right]\right\}^{-1 / 2}, \\
x_{j k n}=w_{j k}^{\prime \prime}\left(s_{j, i_{j 1}}^{\prime \prime} X_{i_{j 1}}^{\prime \prime}+\cdots+s_{j, i_{j q}}^{\prime \prime} X_{i_{j q}}^{\prime \prime}\right)+d_{j k n}, \quad k=1, \ldots, K_{j}, \\
s_{j, i j 1}^{\prime \prime} X_{i_{j 1}}^{\prime \prime}+\cdots+s_{j, i j q}^{\prime \prime} X_{i_{j q}}^{\prime \prime}=\sum_{k}\left(w_{j k}^{\prime \prime} x_{j k n}\right)+d_{j n}, \\
s_{j, i_{j a}}^{\prime \prime}=\operatorname{sgn} r\left(X_{j n}^{\prime \prime} X_{i_{j q}, n}^{\prime \prime}\right), \quad a=1, \ldots, q .
\end{gathered}
$$

To start the iterative procedure with $r=1$ all weights are taken to be equal, say,

$$
w_{j k}^{\prime}=1, \quad j=1, \ldots, J, \quad k=1, \ldots, K_{j}
$$


After the first step, we use the weights $w_{j k}^{\prime}$ obtained in the $r$ th step, together with the linear aggregates shown in (8) and the standardizing factors $f_{j}^{\prime \prime}$ determined by (9), in order to obtain the case values $X_{j n}^{\prime \prime}$ for the LVs in step $(r+1)$. Then, according as the weight relations for the $j$ th block are decided to be mode A or B, the weights $w_{j k}^{\prime \prime}$ in step $(r+1)$ are computed by the simple OLS regressions shown in (10) and (11), respectively. In either case we use the latent variables $X_{j n}^{\prime \prime}$ obtained in (8) and the signs in (12) at the $(r+1)$ th step.

The passage to the limit as $r \rightarrow \infty$ gives the PLS estimates of both the weights and the LVs. Thus the estimated weights and LVs are given by

$$
\begin{aligned}
w_{j k} & =\lim _{r \rightarrow \infty} w_{j k}^{\prime}, \quad X_{j n}=\lim _{r \rightarrow \infty} X_{j n}^{\prime}, \\
j & =1, \ldots, J, \quad k=1, \ldots, K_{j}, \quad n=1, \ldots, N .
\end{aligned}
$$

When the first stage of the PLS algorithm is completed, the second stage uses the LVs estimated in the first stage to estimate the block structure (1.4) by simple OLS regressions and the inner relations $(1.10)$ by simple or multiple OLS regressions, as the case may be. The causal-predictive relations (1.18) are then estimated by substitution.

The third stage of the PLS algorithm estimates the location parameters of the LVs, the block structure, the inner relations, and the causal-predictive relations. In the PLS algorithm the estimation of location parameters is immediate matter, as always in LS estimation.

Equations (2)-(14) for the PLS algorithm with raw data input (1) are readily transformed in order to be able to use data input in the form of cross-product data input. The ensuing parameter estimates are numerically the same except for rounding-off errors. Combined use of the parameter estimates and the raw data then provides estimates of the case values of the LVs and of the residuals in the block structure, the inner relations, and the causal-predictive relations--estimates that are important for the interpretation, testing, and further evolution of the model.

The PLS algorithm is easily implemented on the computer for raw data input as well as for cross-product data input. Computer programs allowing optional use of raw data input or cross-product data input are available in several versions. ${ }^{5}$

${ }^{5}$ The computer program packages MIDAS and MINITAB need no further implementation to run the basic PLS algorithm. In the program of Hui (1978) the PLS procedure is combined with the Fix-Point algorithm to estimate soft models that have interdependencies in the path of inner relations. The program of Apel \& Lohmöller (1980) combines PLS with Stone-Geisser cross-validation for testing the predictive significance of soft modeling. 


\subsection{Three Special Cases of PLS Modeling}

Two PLS models are well-known from multivariate analysis. To begin, the first principal component is numerically equivalent, up to a scale factor, to a one-block PLS model estimated using the mode A procedure. ${ }^{6}$ The classical model standardizes the loadings $\pi_{1 k}$ to unit square sum. The iterative procedure (8)-(12) in this case shrinks to Eqs. (8)-(10) with (10) reducing to

$$
x_{1 k n}=w_{1 k}^{\prime \prime} X_{j n}^{\prime \prime}+d_{1 k n}, \quad k=1, \ldots, K_{1}, \quad n=1, \ldots, N .
$$

The two-block PLS path model estimated using the mode B procedure gives the first canonical coefficient as the estimated correlation between the two latent variables. ${ }^{6}$ The iterative procedure now shrinks to the use of Eqs. (8), (9), and (11), with (11) given by

$$
\begin{array}{ll}
X_{2 k n}^{\prime \prime}=\sum_{k}\left(w_{1 k}^{\prime \prime} x_{1 k n}\right)+d_{1 k n}, & k=1, \ldots, K_{1}, \\
X_{1 k n}^{\prime \prime}=\sum_{k}\left(w_{2 k}^{\prime \prime} x_{2 k n}\right)+d_{2 k n}, & k=1, \ldots, K_{2}, \quad n=1, \ldots, N .
\end{array}
$$

As a third case, consider the Adelman model. The appropriate relations to be used in Adelman's model are mode $\mathrm{C}$ since the model contains both endogenous and exogenous variables. Consequently, the weight relations are formed by mode B for the two first blocks, these being exogenous, and by mode $\mathrm{A}$ for the two last blocks, which are endogenous. The weight relations for Adelman's model are

$$
\begin{aligned}
s_{31} X_{3 n}+s_{41} X_{4 n} & =\sum_{k}\left(w_{1 k} x_{1 k n}\right)+d_{1 n}, \\
s_{32} X_{3 n}+s_{42} X_{4 n} & =\sum_{k}\left(w_{2 k} x_{2 k n}\right)+d_{2 n}, \\
x_{3 k n} & =w_{3 k}\left(s_{31} X_{1 n}+s_{32} X_{2 n}+s_{34} X_{4 n}\right)+d_{3 k n}, \\
x_{4 k n} & =w_{4 k}\left(s_{41} X_{1 n}+s_{42} X_{2 n}+s_{43} X_{3 n}\right)+d_{4 k n},
\end{aligned}
$$

where $n=1, \ldots, 74$ throughout and $k=1, \ldots, K_{j}$ in Eqs. (17)-(20) with $K_{j}=7,13,11$, and 8, respectively.

The second stage of the PLS estimation procedure makes use of the LVs obtained in the first stage to estimate the structural relations. First, the block structure as shown in Eq. (1.4) is estimated by use of an OLS regression of the manifest variables $x_{j k}$ on the estimate $X_{j}$ of the $j$ th LV. The regression

${ }^{6}$ H. Wold (1966). 
equation without the location parameters is as follows:

$$
x_{j k n}=p_{j k} X_{j n}+u_{j k n}, \quad j=1, \ldots, J, \quad k=1, \ldots, K_{j},
$$

giving $p_{j k}$ as the estimate for $\pi_{j k}$ in Eq. (1.4).

In Eq. (9), the sign of the standardization factor $f_{j}^{\prime \prime}$ was left ambiguous. The sign chosen for the limiting factor $f_{j}$ will, by Eq. (8), determine the sign for the array of estimated case values $X_{j n}(n=1, \ldots, N)$ of the latent variable $\xi_{j}$, and hence by Eq. (21) the sign for the array of estimated loadings $p_{j k}\left(k=1, \ldots, K_{j}\right)$. Thus for the $j$ th block, there are two possibilities for choosing the sign of $X_{j}$ and of the array of loadings $p_{j k}$. The model builder should choose that sign for $X_{j}$ which agrees with most of the presumed signs for the theoretical loadings. That is, he should choose that sign so as to give

$$
\sum_{k}\left(\operatorname{sgn} \pi_{j k} p_{j k}\right)>0 \text {. }
$$

However, suppose that the investigator estimates the model in one of two different ways: either, say, by mode $\mathrm{A}$ or mode $\mathrm{C}$. The rule in the previous paragraph should then be applied to determine the signs for the mode $\mathrm{A}$ estimate of $X_{j n}$, say, $X_{j n}(\mathrm{~A})$. For mode C, the sign of $f_{j}$ should thereafter be chosen so as to give positive correlation over $n$ of $X_{j n}(\mathrm{~A})$ and $X_{j n}(\mathrm{C}){ }^{7}$

$$
r\left[X_{j n}(\mathrm{~A}), X_{j n}(\mathrm{C})\right]>0 .
$$

In Adelman's model, the mode $\mathrm{C}$ procedure was chosen. Writing $r_{i j}=r\left(x_{i}, x_{j}\right)$ for the correlations between the estimated LVs, the resulting six values are shown on the first line of Table 2 . For comparison, we also give the corresponding mode A and mode B estimates. Since Adelman's arrow scheme has no arrow between the first two LVs, there is nothing in the

\section{TABLE 2}

Simple Correlations Between LVs Given Alternative Estimation Procedures ${ }^{a}$

\begin{tabular}{lllllll}
\hline & $r_{23}$ & $r_{24}$ & $r_{13}$ & $r_{14}$ & $r_{34}$ & $r_{12}$ \\
\hline Mode C & .766 & .779 & .699 & .797 & .481 & .918 \\
Mode A & .671 & .776 & .705 & .784 & .488 & .912 \\
Mode B & .842 & .889 & .810 & .878 & .709 & .934 \\
\hline
\end{tabular}

${ }^{a}$ Here and in the text the numerical results on Adelman's model draw from Apel \& H. Wold (1980).

${ }^{7}$ H. Wold (1978b). 
PLS estimation procedure that makes for close agreement between the estimates $r_{12}$; hence this column has been placed by itself.

The correlations mode $\mathrm{C}$ and $\mathrm{A}$ show close agreement. Relative to mode $\mathrm{B}$ the differences are larger. The inner relations shown in Eq. (1.10) are estimated without location parameters in terms of the regression equation:

$$
X_{j_{x^{n}}}=b_{\left(j_{*}\right)} X_{\left(j_{x}\right)_{n}}+e_{j_{n}}, \quad j=j_{1}, \ldots, j_{H}, \quad n=1, \ldots, N,
$$

where $b_{\left(j_{x}\right)}$ is the OLS estimate for $\beta_{\left(j_{x}\right.}$. As before using mode $\mathrm{C}$ estimation, the inner relations in Adelman's model are, in this stage, without location parameters:

$$
\begin{array}{ll}
X_{3}=-.026 X_{1}+.790 X_{2}+e_{3}, & \operatorname{var}\left(e_{3}\right)=.413, \\
X_{4}=.514 X_{1}+.517 X_{2}-.274 X_{3}+e_{4}, & \operatorname{var}\left(e_{4}\right)=.319 .
\end{array}
$$

For comparison we give the inner relations when estimated by the mode A procedure,

$$
\begin{array}{ll}
X_{3}=.554 X_{1}+.166 X_{2}+e_{3}, & \operatorname{var}\left(e_{3}\right)=.498, \\
X_{4}=.537 X_{1}+.338 X_{2}-.151 X_{3}+e_{4}, & \operatorname{var}\left(e_{3}\right)=.351 .
\end{array}
$$

We see that for the inner coefficients $b_{\left(j_{*}\right)}$, the difference between modes $A$ and $\mathrm{C}$ is larger than for the simple total correlations $r_{i j}$. The difference in estimation robustness is natural since the $b$ s are partial correlations and are nonlinear functions of the simple correlations $r_{i j}$.

Although the residual variances are somewhat smaller for mode $\mathrm{C}$, which we have chosen for the estimation of Adelman's model, the inner relations estimated by mode $\mathrm{A}$ are more plausible. For example, in mode $\mathrm{C}$ it is against common sense that $X_{1}$, the LV that measures the level of economic development, has a coefficient $b_{31}$ that is insignificant or even negative. Part of the explanation may be the difference in robustness just referred to. Another part of the story is that for the group of 74 countries, the model structure is nonlinear with regard to the level of economic growth.

This last point has been emphasized by Adelman \& Morriss $(1967,1973)$ and can be illustrated in terms of the inner relations as estimated separately for the three groups of countries $(\mathrm{L}, \mathrm{M}, \mathrm{H})$ classified with regard to economic growth; see Fig. 1b. Using the mode $\mathrm{C}$ procedure, the estimated inner relations for the 28 countries with low (L) economic growth are

$$
\begin{aligned}
& X_{3}=.093 X_{1}+.665 X_{2}+e_{3}, \\
& X_{4}=.390 X_{1}+.950 X_{2}-.672 X_{3}+e_{4},
\end{aligned}
$$


and for the 25 countries with high $(\mathrm{H})$ economic growth, they read

$$
\begin{aligned}
& X_{3}=.356 X_{1}+.596 X_{2}+e_{3}, \\
& X_{4}=.159 X_{1}+1.158 X_{2}-.601 X_{3}+e_{4} .
\end{aligned}
$$

The coefficient $b_{31}$ is positive for the intermediate (M) group of 21 countries with a value of .151 .

When the countries are grouped with regard to economic growth, the sample size is only about one third of the total sample. Hence in Eqs. (29) and (30), the estimates of the inner coefficients $b_{(j)}$ are less robust than for the relations in Eqs. (25) and (26) for the entire group of 74 countries. Finally, let us again examine a shift in estimation mode with regard to the robustness of the inner relations. For the countries group $(\mathrm{H})$, the inner relations, when estimated by mode $\mathrm{A}$, are

$$
\begin{aligned}
& X_{3}=.373 X_{1}+.530 X_{2}+e_{3}, \\
& X_{4}=.522 X_{1}-.261 X_{2}+.397 X_{3}+e_{4} .
\end{aligned}
$$

We are now in a position to compare the robustness of the inner coefficients $b$ with regard to the shift in estimation from mode $\mathrm{C}$ to $\mathrm{A}$, on the one hand, and to the nonlinearity of the model structure on the other. It appears that the robustness is more pronounced for the shift in estimation mode than for the shift from total sample to subgroup $(\mathrm{H})$. This is so whether the comparison is based on estimation Mode $\mathrm{C}$ as applied to the total sample or the subgroup $(\mathrm{H})$.

\subsection{The Casual-Predictive Relations}

The relations shown in Eq. (1.18) can be written in terms of the estimated parameters and LVs as

$$
\begin{gathered}
x_{j_{*} k n}=p_{j_{k} k} b_{\left(j_{\star}\right)} X_{\left(j_{\star}\right) n}+v_{j_{t_{k}} n}, \\
j_{*}=j_{1}, \ldots, j_{H}, \quad k=1, \ldots, K_{j_{*}}, \quad n=1, \ldots, N,
\end{gathered}
$$

with prediction error

$$
v_{j_{t} k n}=u_{j k n}+p_{j_{\sharp} k} e_{j k n} .
$$

Alternatively, one can write

$$
x_{j_{\star} k n}=\hat{p}_{j_{*} k} b_{\left(j_{k}\right)} X_{\left(j_{k}\right) n}+\hat{v}_{j_{*} k n},
$$

where the "reestimated loadings" $\hat{p}_{j_{*} k}$ are obtained by computing for fixed $j_{*}, k$ the simple OLS regression of $x_{j_{*} k n}$ on the composite variable $b_{\left(j_{*}\right)} X_{\left(j_{k}\right) n}$, 
a regression over $n=1, \ldots, N$. In virtue of the least squares property of the residuals $\hat{v}_{j_{k} k n}$,

$$
\operatorname{var}\left(\hat{v}_{j_{k} k}\right) \leq \operatorname{var}\left(v_{j_{k}}\right), \quad j_{*}=j_{1}, \ldots, j_{H}, \quad k=1, \ldots, K_{j_{*}} .
$$

Causal-predictive relations in terms of the manifest variables can be obtained from Eqs. (35) or (37) by using (3) to substitute for the right-hand LVs as estimated by the linear aggregates of their indicators. For the reestimated version (37) this gives

$$
x_{j_{\star} k n}=\hat{p}_{j_{\star} k} \sum_{i<j_{\star}}\left[b_{j_{*} i} f_{i} \sum_{n=1}^{K_{1}}\left(w_{i h} x_{i h n}\right)\right]+\hat{v}_{j_{k} k n},
$$

where the residual remains the same as in Eq. (37),

$$
\hat{v}_{j_{k} k n}=u_{j k n}+\hat{p}_{j_{*}} e_{j k n} \text {. }
$$

It will suffice for our purposes to illustrate only the first two versions of the estimation of the causal-predictive relations. The results for the first version as applied to countries $(\mathrm{H})$ are for $j=3, k=3$ and $j=4, k=1$

$$
\begin{aligned}
& x_{33 n}=.313 X_{1 n}+.523 X_{2 n}+v_{33 n}, \quad \frac{1}{11} \sum_{k}\left(\operatorname{var} v_{3 k}\right)=.580 \\
& x_{41 n}=.136 X_{1 n}+.989 X_{2 n}-.514 X_{3 n}+v_{41 n}, \quad \frac{1}{8} \sum_{k}\left(\operatorname{var} v_{4 k}\right)=.796 .
\end{aligned}
$$

The second version with re-estimated loadings is

$$
\begin{aligned}
& x_{33 n}=.287 X_{1 n}+.480 X_{2 n}+\hat{v}_{33 n}, \quad \frac{1}{1 !} \sum_{k}\left(\operatorname{var} \hat{v}_{3 k}\right)=.576, \\
& x_{41 n}=.112 X_{1 n}+.813 X_{2 n}-.422 X_{3 n}+\hat{v}_{41 n}, \quad \frac{1}{8} \sum_{k}\left(\operatorname{var} \hat{v}_{4 k}\right)=.779 .
\end{aligned}
$$

Note that both the residual variances and the coefficients of the LVs decrease from Eqs. (41)-(42) to Eqs. (43)-(44). This is perhaps surprising at first sight but is in perfect accordance with the reestimation of the loadings $p_{j_{*} k}$ in Eq. (37).

\subsection{Estimates OF LOADINGS AND LVS}

In PLS path models the inner relations and the causal-predictive relations give new knowledge of a broad aggregate kind. Knowledge of a more specific kind is given by the estimates $X_{j}, p_{j k}$ of the LVs $\xi_{j}$ and the loadings $\pi_{j k}$ in the block structure.

For the readers of Adelman and Morriss' trail blazing monographs (1967, 1973), the numerical illustrations of the PLS estimation of Adelman's model (1975a), cited in Sections 2.3 and 2.4, cause no surprise, least of all for Adelman 
and Morriss themselves. With factor analysis as their main tool, which they handle with superb skill, Adelman and Morriss classify the 74 countries covered by their data bank into the three groups $(\mathrm{L}, \mathrm{M}, \mathrm{H})$ and perform a penetrating causal analysis of economic growth and equity. The present analysis places more distinct emphasis on cause-effect aspects and provides a quantitative sharpening of some of Adelman-Morriss' arguments. This is so also for the subsequent generalization to LVs in two dimensions.

In coping with the causal-predictive analysis of problems with high complexity and low information, the key feature of soft modeling is the explicit, albeit deliberately approximate, estimation of each LV by a weighted aggregate (1.3) of its indicators. This key feature is reflected in several important aspects of the PLS approach: flexibility, "instant estimation," convergence, partial least squares optimization, and consistency at large. Let us briefly focus on each feature.

\subsection{FLEXIBILITY}

The basic design of PLS "soft modeling" is malleable to the problem under analysis due to the flexibility of the arrow scheme. When the observables are grouped into blocks for indirect measurement of the LVs, the composition, number, and size of the blocks are flexible as well as are the number and structure of the inner relations. The flexibility is heightened by the choice of estimation mode A, B, or C, wherein two LS extremum principles come into play.

More precisely, the first principal component gives LS prediction of the indicators when the LV (the component) is given and is estimated by mode A. The first canonical correlation gives maximum correlation between two LVs and is estimated by mode B. In the passage from two to three or more blocks in the PLS model, the extremum principles of principal components and canonical correlations carry over, albeit in a qualified sense.

When the weight relations of the $j$ th block are estimated by mode A, they are given by Eq. (6) and provide ancillary predictive relations for the observables in the block in terms of estimates of the LVs with which the $j$ th $\mathrm{LV}$ is directly connected in the arrow scheme. By the least squares principle, the prediction errors of the relations shown in (6) have minimum variance. But when the weight relations of the $j$ th block are estimated by mode B procedures, they are given by Eq. (7) and provide estimates for the correlations of the $j$ th LV and the LVs with which the $j$ th LV is directly connected in the arrow scheme. By virtue of the least squares principle, the sum of the absolute values of these intercorrelations will be maximum for given estimates of these last mentioned LVs. 


\section{7. "INSTANT Estimation"}

This description of PLS estimation is suggested because the relations used in the estimation can be written down directly from the arrow scheme and because of the easy and speedy computer work.

Two features in the PLS approach are instrumental in making for instant estimation; namely, the explicit estimation of the LVs by the use of weighted aggregates of their indicators and the direct estimation of the weight relations by mode A or B. Thanks to the explicit estimation of the LVs, no identification problems arise in "soft" modeling. The weight relations avoid the nonlinear side relations that would arise if the aggregate relations were treated as side conditions and had to be taken into account through Lagrange multipliers. However, the weight relations are not deduced from the PLS model as defined by the block structure and the inner relations but are ancillary information gathered from the intercorrelations between each LV and its adjacent $L V s$ in the arrow scheme.

\subsection{CONVERGENCE}

For one- and two-block models the PLS estimation procedure converges almost certainly. ${ }^{8}$ For PLS models with three or more blocks, convergence of the estimation procedure has never been a problem in applications to real-world models and data. For example, in the numerical estimation of Adelman's model, convergence of the iterative procedure was reached for modes $\mathrm{A}, \mathrm{B}$, and $\mathrm{C}$ in three, six, and four steps, respectively.

\subsection{PLS Optimization}

In PLS estimation, each regression step of the procedure is an LS estimation of one or more unknowns in terms of proxy or final estimates for one or more other unknowns; no total, or overall, extremum condition is imposed as a unifying condition.

The PLS procedure minimizes in its first stage each residual variance in the weight relations that for the various blocks of the model are given by Eqs. (6) or (7). This is done for each block depending on the choice of estimation mode A or B for that block. In each step $r=1,2, \ldots$ the iterative first stage of the PLS algorithm is nothing other than the conventional LS

${ }^{8}$ Lyttkens, Areskoug \& H. Wold (1975). 
procedure, the residual variance of a weight relation being minimized with regard to the free parameters which are the weights. Mode A uses simple OLS regressions to minimize with respect to the parameters one by one, whereas mode B uses multiple OLS regression to minimize with regard to the parameters jointly. The conformity with the conventional LS procedure carries over to the limit as $r \rightarrow \infty$.

The second stage of the PLS algorithm uses the LVs obtained in the first stage to estimate the parameters of the structural relations of the model. This is performed by minimizing the residual variances in the block structure, the inner relations, and the causal-predictive relations SELV with reestimation. Thus the PLS procedure is partial LS in the sense that each step of the procedure minimizes a residual variance with respect to a subset of the free parameters, given proxy or final estimates for the other parameters. In the limit the PLS estimation procedure is coherent in the sense that all the residual variances are minimized jointly. However, the PLS procedure remains partial in the sense that no total residual variance or other overall criterion is set up for optimization. ${ }^{9}$

\subsection{CONSISTENCY AND CONSISTENCY AT LARGE}

These limit properties of PLS estimation refer to the passage to the limit as the sample size increases (consistency) and as the block size increases as well (consistency at large). Both properties hold good for PLS estimation under mild supplementary conditions to the model structure defined in Eqs. (1.3)-(1.11). However, consistency does not imply that the estimator is unbiased in the limit; a well-known example is the canonical correlation coefficient. While consistency is a simple corollary of predictor specification, see Footnote 4, consistency at large is a straightforward implication of the classical probability theorem known as the Law of Large Numbers. ${ }^{10}$

\section{Construction and Evaluation of PLS Models}

The PLS approach to path models with LVs has developed gradually since 1971, as documented in a series of progress reports, and it is even more recently that its foundations have been consolidated enough to support the crucial transition from a pioneering device to a useful tool for applied work. Table 3 refers to some 20 applications to real-world problems and data that

${ }^{9} \mathrm{H}$. Wold (1980b).

${ }^{10} \mathrm{H}$. Wold (1978a, 1980b). 


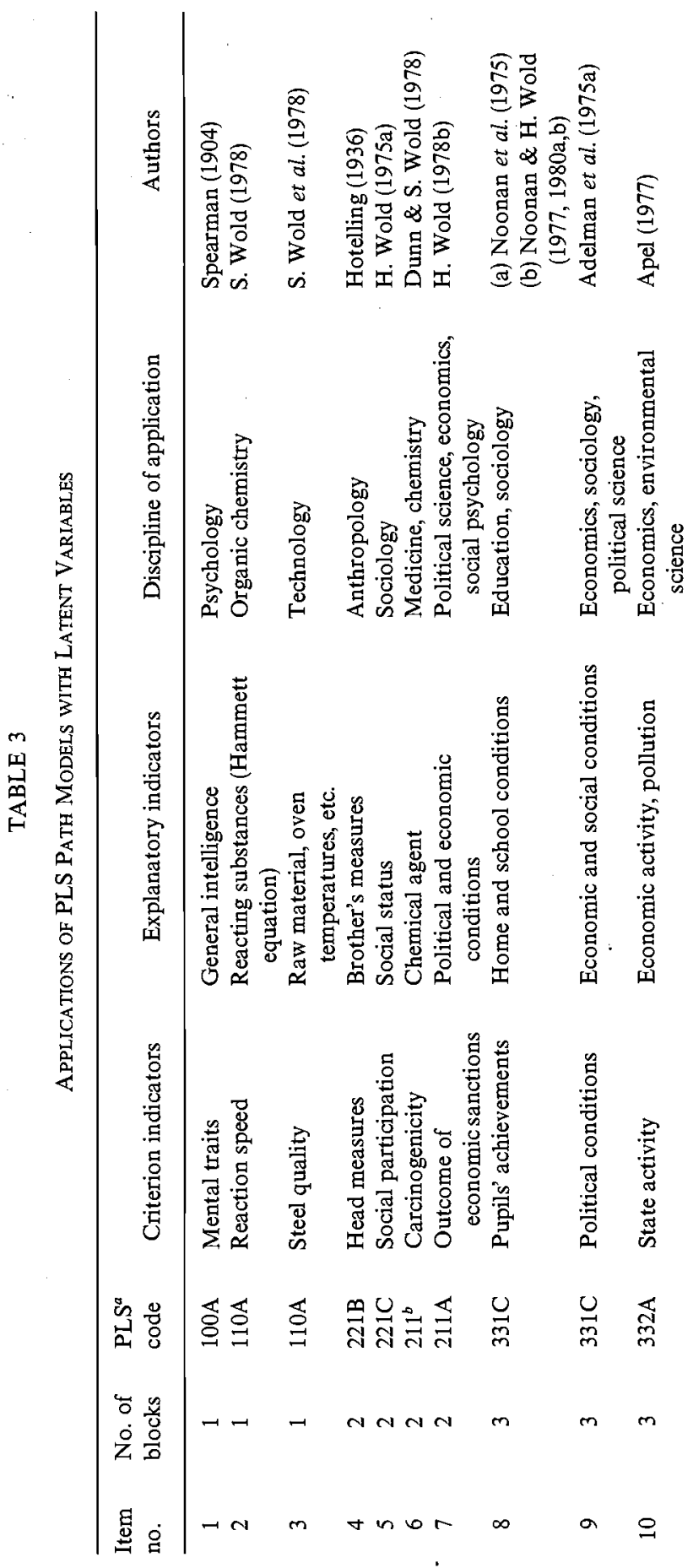




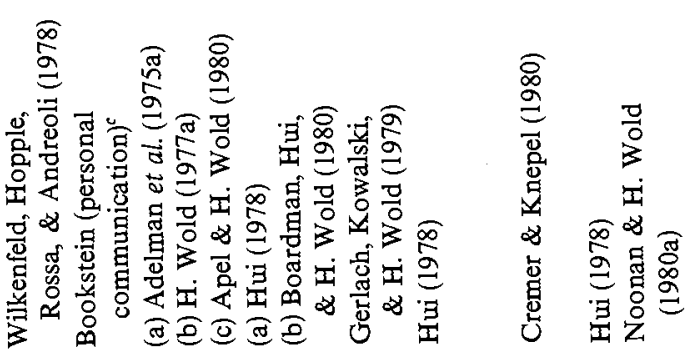

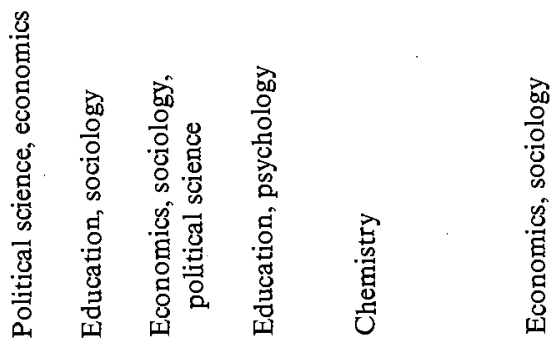

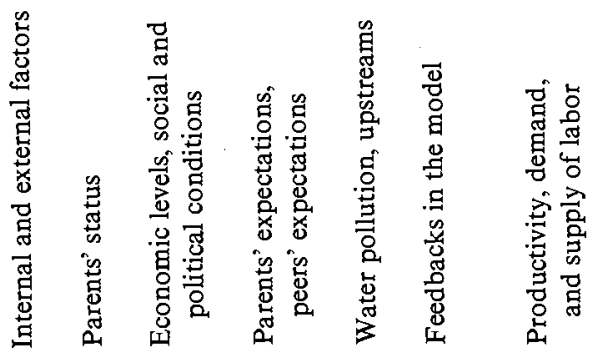

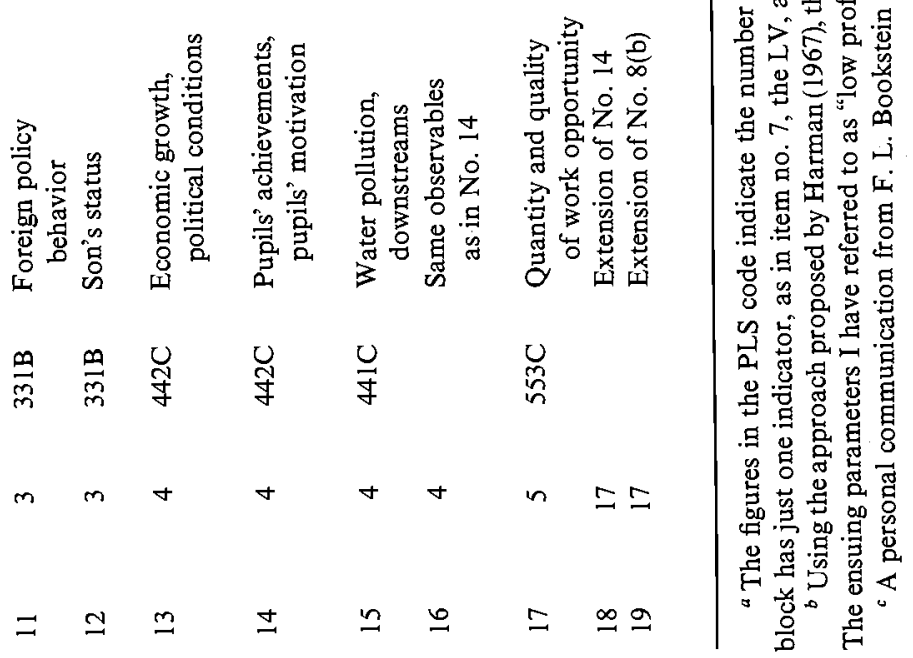


were available when this chapter was drafted. The dates in the last column show that the PLS approach is gaining momentum but is still in an early stage of evolution. The purpose of this final section is to summarize the evolution of PLS model construction and to discuss the accumulating experience about the scope and limitations of the PLS approach.

\subsection{When to, How to, AND Why to USE PLS}

As stated at the outset PLS modeling is primarily designed for causalpredictive analysis of problems with high complexity, but low information. Table 3 gives evidence of the wide applicability of PLS modeling with latent variables. Complex problems with low information occur in most or all sciences and the list includes examples ranging from the natural and medical to the social and political sciences.

Being distribution-free, PLS estimation imposes no restrictions on the format or the data. The available data may be time series or cross sections. The observations on the indicators may be quantitative measurements, ordinal ranks, or records of occurrence, nonoccurrence, or of low versus high levels of the indicator.

The arrow scheme is the conceptual-theoretical blueprint for the model. The arrow scheme indicates which LVs enter the model, which observablesindicators are to be used and their grouping into the blocks for indirect measurement of the LVs, and which "inner relations" are assumed to exist between the LVs. Speaking broadly, the researcher designs the arrow scheme on the joint basis of his rudimentary theoretical knowledge, his experience and intuition about the problems explored, and the data that are at his disposal.

The arrow scheme is usually tentative since the model construction is an evolutionary process. The empirical content of the model is extracted from the data, and the model is improved by interactions through the estimation procedure between the model and the data and the reactions of the researcher. Consequently, the researcher should begin with a generous number of observables-indicators in the various blocks. To use many observables makes for rich empirical content of the model and is favorable to the accuracy of the PLS estimation procedure. In the interaction between the data and the original model it will become apparent which indicators are relevant and which should be omitted. The researcher in planning his empirical analysis must choose between the various modes of estimation. To use estimation mode $\mathrm{A}$ for the whole model gives minimum residual variances in the block structure; but to use mode B for the whole model leads to maximum intercorrelations between the LVs that are directly connected in the arrow scheme. The hybrid mode $\mathrm{C}$ strikes a balance in these respects. 
In the cause-effect and predictive performance of a soft model the relevance of the various LVs hinges upon their indicators. In the relationship between an LV and its indicators the model gives two measures for the relevance of an indicator, namely, its loading and its weight. The difference is analogous to the difference between the coefficients from simple and multiple regression. Thus for the indicator $x_{j k}$, its loading $p_{j k}$ measures the separate or individual contribution of $x_{j k}$ to the relevance of the LV of the $j$ th block, whereas its weight $w_{j k}$ measures the contribution of $x_{j k}$ to the joint relevance of the indicators in the $j$ th block and thereby to the relevance of the $j$ th LV. Both measures are of importance; hence in applied work with soft modeling both measures should be used and reported.

The causal-predictive accuracy of a specific explanatory variable and its coefficient, on the assumption that the other variables remain constant, hinges upon whether the selection of explanatory variables is sufficiently realistic and complete. For example, a dormant variable that comes into play may bring drastic changes in the coefficients.

As to the distribution between causal and predictive inferences, causal relations are always predictive, but predictive relations are not necessarily causal. The question whether an explanatory variable is not only predictive, but also causal, belongs to the subject matter of the model.

\section{ACKNOWLEDGMENTS}

My research for this paper has been supported by the Stiftung Volkswagenwerk. My thanks and appreciation are due to Dr. Heino Apel for collaboration on Adelman's model and for the numerical results reported in Section 2.3. I would like to express my deep gratitude to Professors Kmenta and Ramsey for inviting me to include in their book an exposition of soft modeling based on Cahier 1979:06, Department of Econometrics, University of Geneva, and to Professor Ramsey for editing an abridgement of Cahier 1979:06 for this purpose.

\section{REFERENCES}

Adelman, I., \& Morriss, C. T. Society, politics, and economic development: A quantitative approach. Baltimore, Md.: Johns Hopkins Press, 1967. Revised edition 1971.

Adelman, I., \& Morriss, C. T. Econonic growth and social equity in developing countries. Stanford, Cal. : Stanford Univ. Press, 1973.

Adelman, I., et al. Applications of classification and path models with latent variables to Adelman-Morriss' data. In H. Wold (Ed.), Group report: Modeling in complex situations with soft information. Toronto: Third world congress of econometrics, August 2l-26, 1975. Research Report 1975:4, University Institute of Statistics, Uppsala, 1975. Chapter 4.(a)

Adelman, I., et al. Perturbation analysis of NIPALS parameter estimates. In H. Wold (Ed.), Group report: Modeling in complex situations with soft information. Toronto: Third world congress of econometrics, August 21-26, 1975. Research Report 1975:4, University Institute of Statistics, Uppsala, 1975. Chapter 6. (b) 
Apel, H. Simulation sozio-ökonomischer Zusammenhänge: Kritik und Modifikation von Systems Analysis. Doctoral dissertation, J. W. von Goethe University, Frankfurt/Main, 1977: Published, Darmstadt: S. Toeche-Mittler, 1979.

Apel, H., \& Lohmöller, J. B. Strukturgleichungsmodelle under Partialeinstquadratkriterien: Darstellung und Beschreibung des Programms PLSX. Unpublished manuscript, Department of Education, Hochschule der Bundeswehr, Munich, 1980.

Apel, H., \& Wold, H. Soft modeling: Latent variables in higher dimensions; cross-validation by Stone-Geisser's test. In K. G. Jöreskog \& H. Wold (Eds.), Proceedings of the conference "Systems under indirect observation. Causality-structure-prediction," October 18-20, 1979, Cartigny near Geneva. Amsterdam: North-Holland Publ., 1980, in press.

Areskoug, B. Properties of partial least squares estimation, and comparison with maximum likelihood. Forthcoming doctoral dissertation, University of Göteborg, 1980.

Blalock, H. M., Jr. Causal inferences in nonexperimental research. Chapel Hill, N. C.: University of North Carolina Press, 1964.

Blalock, H. M., Jr. Causal models in volving unmeasured variables in stimulus-response situations. In H. M. Blalock, Jr. (Ed.), Causal Models in the Social Sciences. Chicago, Ill.: Aldine-A therton, 1971. Pp. 307-357.

Blalock, H. M., Jr., Borodkin, F. M., Boudon, R., \& Capecchi, V. (Eds.) Quantitative sociology. New York: Seminar Press, 1975.

Boardman, A. E., Hui, B. S., Wold, H. The partial least squares-fix point method of estimating in terdependent systems with latent variables. Communications of Statistics, 1980, in press.

Cremer, R., \& Knepel, H. Ein Indikatormodell für sozioökonomische Problembereiche des Arbeitsmarktes. Mitteilungen aus der Arbeitsmarkt-und Berufsforschung, 1980, 13, 125-136.

Duncan, O. D. Path analysis: Sociological examples. American Journal of Sociology, 1966, 72, $1-16$.

Dunn, W. J., III, \& Wold, S. "A structure-carcinogenicity study of 4-nitroquinoline-l-oxides using the SIMCA method of pattern recognition." Joumal of Medical Chemistry, 1978, 68, $1001-1010$.

Geisser, S. A predictive approach to the random effect model. Biometrika, 1974, 61, 101-107.

Gerlach, R. W., Kowalski B. R., \& Wold, H. Partial least squares path modelling with latent variables. Analytica Chinica Acia, 1979, 112, 417-421.

Harman, H. H. Modern factor analysis. Chicago, Ill.: University of Chicago Press, 1967.

Hotelling, H. Relations between two sets of variates. Biometrika, 1936, 28, 321-377.

Hui, B. S. The partial least squares approach to path models of indirectly observed variables with multiple indicators. Doctoral dissertation, University of Pennsylvania, 1978.

Jöreskog, K. G. A general method for analysis of covariance structures. Biometrika, 1970. 57, 239-257.

Jöreskog, K. G. A general method for estimating a linear structural equation system. In A. S. Goldberger \& O. D. Duncan (Eds.), Structural equation systems in the social sciences. New York: Seminar Press, 1973. Pp. 85-112.

Jöreskog, K. G. Structural analysis of covariance and correlation matrices. Psychometric Society Presidential Address, Research Report 78:10, Department of Statistics, University of Uppsala, 1978.

Jöreskog, K. G. The LISREL approach to causal model building in the social sciences. In K. G. Jöreskog \& H. Wold (Eds.), Proceedings of the conference "Systems under indirect observation. Causality-structure-prediction" October 18-20,1979, Cartigny near Geneva. Amsterdam: North-Holland Publ., 1980, in press.

Jöreskog, K. G., \& Goldberger, A. Estimation of a model with multiple indicators and multiple causes of a single latent variable. Journal of the American Statistical Association, 1975, 70, $631-639$. 
Jöreskog, K. G., \& Wold, H. The ML and PLS techniques for modeling with latent variables: Comparative aspects. In K. G. Jöreskog \& H. Wold (Eds.), Proceedings of the conference "Systems under indirect observation. Causality-structure-prediction," October 18-20, 1979, Cartigny near Geneva. Amsterdam: North-Holland Publ., 1980, in press. (a)

Jöreskog, K. G., \& Wold, H. (Eds.), Proceedings of the conference "Systems under indirect. onservation. Casuality-structure-prediction," October 18-20, 1979, Cartigny near Geneva. Amsterdam: North-Holland Publ., 1980, in press. (b)

Lyttkens, E., Areskoug, B., \& Wold, H. The convergence of NIPALS estimation procedures for six path models with one or two latent variables. Research Report 1975:3, Department of Statistics, University of Göteborg, 1975.

Mosbaek, E. J., \& Wold, H., with contributions by E. Lyttkens, A. Agren and L. Bodin. Interdependent systems: Structure and estimation. Amsterdam: North-Holland Publ., 1970.

Noonan, R., et al. Applications of path models with latent variables to the I.E.A. data bank. In $\mathrm{H}$. Wold (Ed.), Group report: Modeling in complex situations with soft information. Toronto: Third world congress of econometrics, August 21-26, 1975. Research Report 1975:4, University Institute of Statistics, Uppsala, 1975. Chapter 5.

Noonan, R., \& Wold, H. NIPALS path modeling with latent variables: Analyzing school survey data using nonlinear iterative partial least squares. Part I. Scandinavian Journal of Educational Research, 1977, 21, 33-61.

Noonan, R., \& Wold, H. NIPALS path modeling with latent variables: Analyzing school survey data using nonlinear iterative partial least squares. Part II. Scandinavian Journal of Educational Research, 1980, 24, 1-24. (a)

Noonan, R., \& Wold, H. NIPALS path modeling with latent variables: Analyzing school survey data using nonlinear iterative partial least squares. Part III. In K. G. Jöreskog \& H. Wold (Eds.), Proceedings of the conference "Systems under indirect observation. Causality-structure-prediction," October 18-20, 1979, Cartigny near Geneva. Amsterdam: North-Holland Publ., 1980, in press. (b)

Spearman, C. General intelligence objectively determined and measured. American Journal of Psychology, 1904, 15, 201-293.

Stone, M. Cross-validatory choice and assessment of statistical predictions. Journal of the Royal Statistical Society, Series B, 1974, 36, 111-133.

Wilkenfeld, J., Hopple, G. W., Rossa, P. J., \& Andriole, S. J. Final report of the interstate behavior analysis project. Washington D.C.: Cybernetics Technology, Office of Naval Research, 1978.

Wold, H. On the consistency of least squares regression. Sankhyā, Series A, 25(2), 1963, 211-215.

Wold, H. Nonlinear estimation by iterative least squares procedures. In F. N. David (Ed.), Research papers in statistics, Festschrift for J. Neyman. New York: Wiley, 1966. Pp. 411-444.

Wold, H. Casual flows with latent variables: Partings of the ways in the light of NIPALS modeling. European Economic Review. Special Issue in Honour of Ragnar Frisch, 1974, $5(1), 67-86$.

Wold, H. Path models with one or two latent variables. In H. M. Blalock Jr., F. M. Borodkin, R. Boudon, \& V. Capecchi (Eds.), Quantitative sociology. New York: Seminar Press, 1975. Pp. 307-357. (a)

Wold, $H$. Soft modelling by latent variables: The non-linear iterative partial least squares (NIPALS) approach. In J. Gani (Ed.), Perspectives in Probability and Statistics, Papers in Honour of M. S. Bartlett. London: Academic Press, 1975. Pp. 117-142. (b)

Wold, $\mathrm{H}$. From hard to soft modeling. In $\mathrm{H}$. Wold (Ed.), Group report: Modeling in complex situations with soft information. Toronto: Third world congress of econometrics, August 21-26, 1975. Research Report 1975:4, University Institute of Statistics, Uppsala, 1975. Chapter 1. (c) 
Wold, H. (Ed.) Group report: Modeling in complex situations with soft information. Toronto: Third world congress of econometrics, August 21-26, 1975. Research Report 1975:4, University Institute of Statistics, Uppsala, 1975. (d)

Wold, $\mathrm{H}$. On the transition from pattern recognition to model building. In R. Henn and $\mathrm{O}$. Moeschlin (Eds.), Mathematical economics and game theory: Essays in honor of Oskar Morgenstern. Berlin: Springer, 1977. Pp. 536-549. (a)

Wold, H. Open path models with latent variables. In H. Albach, E. Helmstedter and R. Henn (Eds.), Kvantitative Wirtschaftsforschung. Wilhelm Krelle zum 60. Geburstag. Tübingen: Mohr, 1977.(b)

Wold, H. Ways and means of interdisciplinary studies. In Transactions of the Sixth International Conference on the Unity of the Sciences, San Francisco, 1977. New York: The International Cultural Foundation, 1978. Pp. 1071-1095. (a)

Wold, H. Factors influencing the outcome of economic sanctions: Tentative analysis by PLS (Partial Least Squares) modeling. University of Haifa: International Workshop of Conflict Resolution, June 19-24, 1978. (b)

Wold, H. (Ed.). The fix-point approach to interdependent systems. Amsterdam: North-Holland Publ., 1980.(a)

Wold, H. Soft modeling: The basic design and some extensions. In K. G. Jöreskog \& H. Wold (Eds.), Proceedings of the conference "Systems under indirect observation. Causality-structure-prediction," October 18-20, 1979, Cartigny near Geneva. Amsterdam: NorthHolland Publ., 1980, in press. (b)

Wold, S. Cross-validatory estimation of the number of components in factor and principal components models. Technometrics, 1978, 20, 397-405.

Wold, S., et al. Four levels of pattern recognition. Analytica Chimica Acta, 1978, 103, 429-443.

Wold, S., et al. The indirect observation of molecular chemical systems. In K. G. Jöreskog \& H. Wold (Eds.), Proceedings of the conference "Systems under indirect observation, Causality-structure-prediction," October 18-20, 1979, Cartigny near Geneva. Amsterdam: North-Holland Publ., 1980, in press. 